\title{
Decolorization of Digital Pathology Images: A Comparative Study
}

\author{
Krishna Gopal Dhal ${ }^{1}$, Swarnajit Ray $^{2}$, Arunita Das ${ }^{3}$, Iztok Fister Jr. ${ }^{4}$, Sanjoy Das ${ }^{5}$ \\ ${ }^{1}$ Dept. of Computer Science and Application, Midnapore College (Autonomous), Paschim Medinipur, West Bengal, India. \\ Email: krishnagopal.dhal@midnaporecollege.ac.in \\ ${ }^{2}$ Learningmate Solutions Pvt. Ltd., Kolkata, West Bengal, India. Email: swarnajit32@gmail.com \\ ${ }^{3}$ Dept. of Information Technology, Kalyani Govt. Engineering College, Kalyani, Nadia, India. Email: arunita17@gmail.com. \\ ${ }^{4}$ Faculty of Electrical Eng. and Computer Sc., University of Maribor, Slovenia, Email: iztok.fister1@um.si. \\ ${ }^{5}$ Dept. of Eng. and Technological Studies, University of Kalyani, Kalyani, India, Email: dassanjoy0810@hotmail.com
}

\begin{abstract}
The major demerit of color to gray conversion is the loss of visually important image features. Digital pathology images are treated as the gold Standard for detection of various diseases, especially for the different types of cancer. Digital pathology images are color in nature, i.e. each pixel is a color vector represented by three values. Thus, the processing of these images requires high computational time. If these color images are converted into one dimensional gray images, then processing time can be reduced, which will help the Computer-Aided Diagnosis (CAD) system significantly for accurate classification and detection of different types of diseases. Therefore, this study focuses on the fast conversion of color digital pathology images into gray images. In order to do that, seven well established color to gray conversion, techniques have been employed for producing gray images with salient features. The outcomes have been validated visually and numerically.
\end{abstract}

\section{KEYWORDS}

Digital Pathology Images, Decolorization, Color to Gray Conversion, Gray Scale, RGB2GRAY.

\section{Introduction}

Computer assisted pathology and microscopy image analysis, assist the decision making for automated disease diagnosing, as they provide digital images related to certain kinds of disease using Computer-Aided Diagnosis (CAD) systems, which facilitates quantitative and qualitative medical results with a high throughput processing rate $[1,2,3]$. At present, automated medical diagnosing has attracted the attention of several pathologists in research and clinical practice, since CAD systems reduce human error, false positive results and time complexity, while pathology imaging provides more accurate results, faster and reproducible image analysis. Digital pathology images are stored as high-resolution color images, i.e. each pixel is represented as a three-dimensional vector, namely $\mathrm{R}, \mathrm{G}$, and $\mathrm{B}$, and, due to that, they are of the order $\mathrm{M} \times \mathrm{N} \times 3$, where $\mathrm{M}$ and $\mathrm{N}$ indicate the number of row and column respectively. Therefore, several image processing techniques, like enhancement, segmentation, require high computational effort. In order to overcome this issue, if these high dimensional images can be reduced to the order $\mathrm{M} \times \mathrm{N}$ with each pixel as a single scalar value, then the computation for applying these techniques reduces drastically. Another benefit is that this conversion facilitates the application of single-channel algorithms on color images, like Canny operator for edge detection [4]. In literature, this dimension reduction is considered as color to gray scale image conversion, or decolorization.

Several color to gray scale conversion techniques have been developed by following the human perception of brightness and contrast, and they proved their efficiency in the traditional color image decolorization field [5-12]. However, the utilization of decolorization techniques in the Digital Pathology domain is a little bit different. Information loss minimization for a specific image is the main aspiration. Therefore, this study utilizes these developed color to gray conversion techniques for the decolorization of pathology images to prove their efficacy in this medical image domain. All color to gray conversion techniques are categorized into three classes, namely Local, Global, and Hybrid. In local processing based techniques [5, 6], the same color pixel within an image can be mapped into different gray values, depending on the local distributions of colors, which is generally not desired. Compared to local, global processing methods [4, 7-12] are able to produce natural looking images. Several hybrid methods have also been developed by considering global and local contrast or features for conversion [13, 14], but, it is also true that local processing and utilization of local information based statistics take large computational time. Therefore, this letter considers only global processing based techniques [4, 7-12], which are discussed in the next Section.

The paper is organized as follows: Section 2 discusses all the global color to gray conversion techniques. Section 3 describes the experimental results and the paper is concluded in section 4 .

\section{Decolorization Models}

The existing global decolorization, methods have been presented in this Section.

\subsection{MATLAB Procedure (rgb2gray)}


Generally, global mapping techniques convert a color image $(R G B)$ into a grayscale image $(G)$ by a linear weighting of the $R$, $G$, and $B$ channels, i.e. $\mathrm{G}(\mathrm{i}, \mathrm{j})=\sum_{\mathrm{c}=\mathrm{R}, \mathrm{G}, \mathrm{B}} w_{\mathrm{c}} R G B_{\mathrm{c}}(\mathrm{i}, \mathrm{j})$, where $\sum_{\mathrm{c}=\mathrm{R}, \mathrm{G}, \mathrm{B}} w_{c}=1$. Here, the three linear weighting parameters $\mathrm{w}$, should be estimated on the basis of some models.

In the MATLAB (Matrix Laboratory) software, developed by MathWorks [11], an RGB image converts into gray-scale by the following weighting formula:

$G=0.2989 \times R+0.5870 \times G+0.1140 \times B$

\subsection{Color2Gray}

This decolorization model was developed by Gooch et. al. in 2005 [7]. The proposed model uses CIELAB color space, and maintains color contrast between pixel pairs by optimizing an objective contrast function.

\subsection{Real-Time Contrast Preserving Decolorization (RTCPD)}

It has previously been said that $\mathrm{G}(\mathrm{i}, \mathrm{j})=\sum_{\mathrm{c}=\mathrm{R}, \mathrm{G}, \mathrm{B}} w_{\mathrm{c}} R G B_{\mathrm{c}}(\mathrm{i}, \mathrm{j})$. In 2009, Lu et. al. [8] also developed a decolorization model called Real-Time Contrast Preserving Decolorization (RTCPD) by optimizing the linear weights $w_{\mathrm{c}}$ by minimizing the gradient error energy function.

\subsection{Gradient Correlation Similarity for Decolorization (GesDecolor)}

The GcsDecolor [9] model was proposed by Liu et. al. in 2015, which is the variant of RTCPD. Gradient correlation similarity (Gcs) measure were utilized in GcsDecolor. Two variants of GcsDecolor have been developed by the authors. The first one is iterative GcsDecolor and the other is discrete searching GcsDecolor. Discrete searching based GcsDecolor is utilized here, due to its simplicity and run time efficiency.

\subsection{Semi-Parametric (SPDecolor) model}

\section{Decolorization}

This Semi-Parametric Decolorization technique is another variant of RTCPD proposed by Liu et. al. in 2016 [4]. SPDecolor has the strength of the parametric contrast preserving method and the non-parametric rgb2gray method.

\subsection{Color to Gray Conversion by Correlation (CorrC2G)}

The CorrC2G [10] technique was proposed by Nafchi et. al. in 2017, where the linear weighting parameters (w) have been estimated using the correlation information between each band of RGB image and a contrast image. This method also does not require any edge information or any optimization.

\subsection{Parametric ratio-based method for efficient contrast decolorization (PrDecolor) preserving}

This PrDecolor was proposed by Xiong et. al. in 2018 [12]. The method is a contrast preserving multivariate parametrical constraint based decolorization model.

\section{Experimental Results}

The experiment was performed over 40 color hematopathology and histopathology images with MatlabR2016a and a Windows10 OS, x64-based PC, RIZEN CPU, $3.6 \mathrm{GHz}$ with 8 GB RAM. The proposed methods were tested on images taken from the ALL IDB dataset [15] and UCSB Bio-Segmentation Benchmark dataset $[16,17]$.

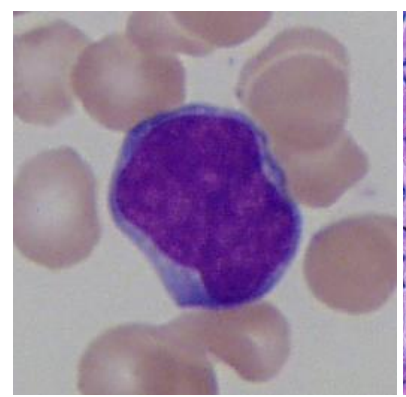

(a)

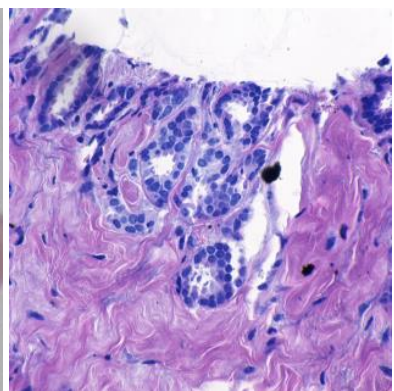

(b)
Fig.1. (a) Original image of Acute Lymphoblastic Leukemia (b) Original image of Breast histopathology

The decolorization efficacy of the proposed models has been judged by computing three quality parameters, namely the Colorto-Gray Structural Similarity (C2G-SSIM) index (C2G-SSIM) [10, 20], Edge based Contrast Measure (EBCM) [18], and Entropy [19]. C2G-SSIM [10, 20] is a color to gray evaluation metric based on the popular image quality assessment metric SSIM. It demonstrates higher correlation with human subjective evaluations. It is expected that the efficient color to gray conversion technique preserves the edge information. Therefore, EBCM has been utilized to measure the edge information, as it is less sensitive to digitization effects and noise. Entropy [19] value reveals the information content in the image. If the distribution of the intensities is uniform, then it can be said that a histogram is equalized and the entropy of the image is more.

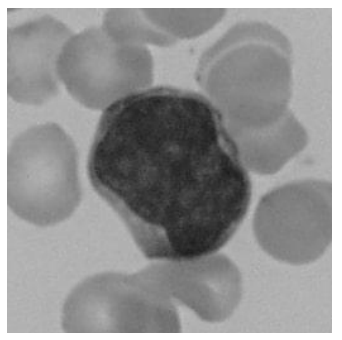

(a)

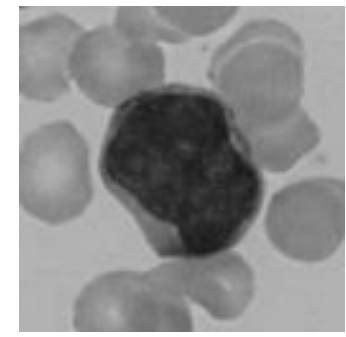

(b) 


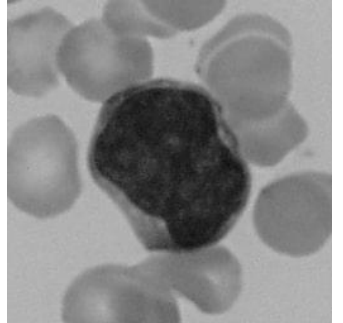

(c)

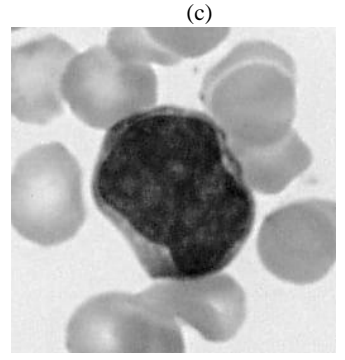

(e)

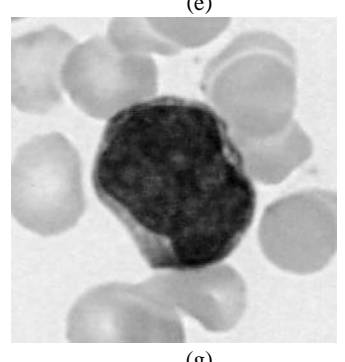

(g)

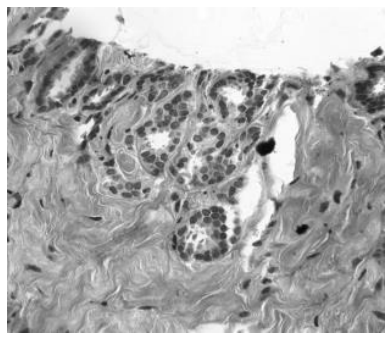

(a)

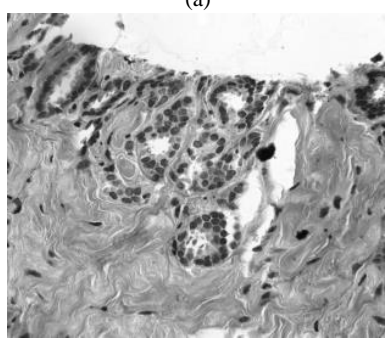

(c)

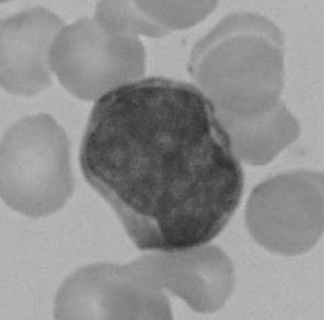

(d)

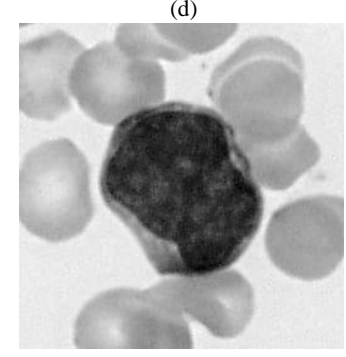

Fig.2. Results of decolorizarion for Fig.1(a): (a) rgb2gray [11] (b) Color2Gray [7] (c) RTCPD [8] (d) GesDecolor [9] (e) SPDecolor [4] (f) CorrC2G [10] (g) PrDecolor [12].

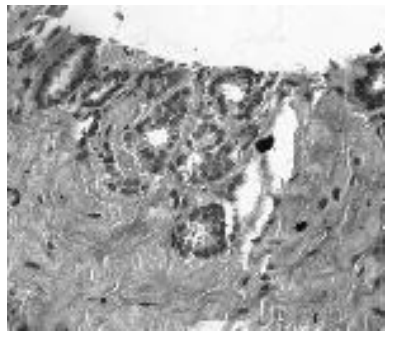

(b)

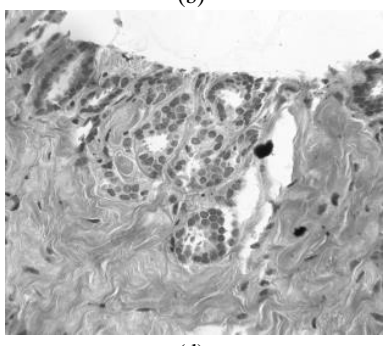

(d)

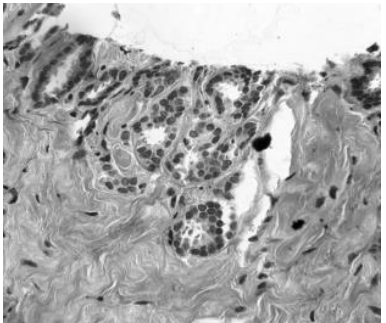

(e)

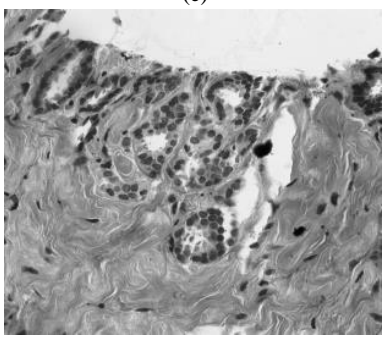

(g)

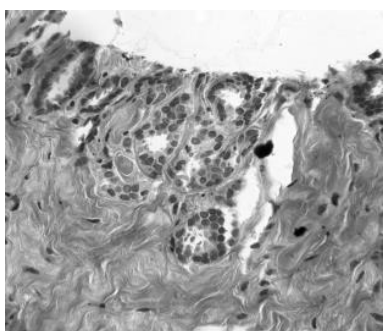

(f) for Fig.1(b): (a) rgb2gray [11] (b) Color2Gray [7] (c) RTCPD [8] (d) GcsDecolor [9] (e) SPDecolor [4] (f) CorrC2G [10] (g) PrDecolor [12].
Fig.3. Results of decolorizarion

Table 1. Average Quality parameters over 40 images

\begin{tabular}{|l|c|l|l|}
\hline \multicolumn{1}{|c|}{ Method } & C2G-SSIM & EBCM & \multicolumn{1}{c|}{ Entropy } \\
\hline rgb2gray [11] & 0.8912 & 183.24 & 7.19 \\
\hline Color2Gray [7] & 0.8314 & 172.13 & 6.98 \\
\hline RTCPD [8] & 0.8914 & 183.57 & 7.19 \\
\hline GcsDecolor [9] & 0.8598 & 174.90 & 7.11 \\
\hline SPDecolor [4] & 0.9030 & 187.38 & 7.23 \\
\hline CorrC2G [10] & 0.9032 & 187.98 & $\mathbf{7 . 2 5}$ \\
\hline PrDecolor [12] & $\mathbf{0 . 9 0 3 5}$ & $\mathbf{1 8 8 . 7 4}$ & $\mathbf{7 . 2 5}$ \\
\hline
\end{tabular}

/* Best results obtained are given in bold*/

Table 2. Computational time of decolorization methods

\begin{tabular}{|l|c|l|}
\hline \multicolumn{1}{|c|}{ Method } & $\begin{array}{c}\text { Fig.1(a) } \\
\text { 257x257 }\end{array}$ & $\begin{array}{c}\text { Fig.1(b) } \\
896 \times 768\end{array}$ \\
\hline rgb2gray [11] & $\mathbf{0 . 0 0 8 6}$ & $\mathbf{0 . 0 2 2 1}$ \\
\hline Color2Gray [7] & 157.01 & 263.23 \\
\hline RTCPD [8] & 0.0721 & 0.0636 \\
\hline GcsDecolor [9] & 0.0397 & 0.0723 \\
\hline SPDecolor [4] & 0.0942 & 1.0967 \\
\hline CorrC2G [10] & 0.0187 & 0.0385 \\
\hline PrDecolor [12] & 2.9678 & 27.8316 \\
\hline
\end{tabular}

/* Best results obtained are given in bold*/

\subsection{Analysis of Experimental Results}

Performance analysis of the considered seven decolorization models was performed by using three image quality parameters and computational time. Figs. 2 and 3 demonstrate the outcomes of the seven decolorization models over pathology images, represented as Fig. 1. Values of the quality parameters and computational times are given in Tables 1 and 2 respectively. Visual analysis of Figs. 2 and 3 shows clearly that SPDecolor [4], CorrC2G [10], and PrDecolor [12] produce better outcomes compared to other decolorization methods. However, when we compare these methods based on quality parameters, it can be seen that PrDecolor [12] outperforms the other methods. 
SPDecolor [4] and CorrC2G [10] provide nearly the same results as PrDecolor [12], as their corresponding numerical values of the quality parameters are almost the same. It can also be seen that these three methods, namely, SPDecolor [4], CorrC2G [10], and PrDecolor [12] outperform the other four methods significantly in terms of quality parameters. When we consider computational time, it can be seen that the MATLAB based rgb2gray [11] method is the best method. However, among the SPDecolor [4], CorrC2G [10], and PrDecolor [12] methods, CorrC2G is associated with the lowest computational time.

\section{Conclusion}

This paper presents a comparative study among seven existing decolorization methods in the case of digital pathology images. The visual and decolorization quality parameters prove clearly that PrDecolor [12], proposed by Xiong et. al., provided the best outcomes compared to the other six methods. Computational time shows that the MATLAB based rgb2gray method outperformed the others, although CorrC2G [10] produced nearly the same outputs as the PrDecolor [12] method, but within the second less computational time. One challenging future direction of this study can be the application of nature-inspired optimization algorithms to set the parameters of the parametric decolorization methods by considering different objective functions.

\section{REFERENCES}

1) Gurcan, M. N., Boucheron, L. E., Can, A., Madabhushi, A., Rajpoot, N. M., \& Yener, B. (2009). Histopathological image analysis: A review. IEEE reviews in biomedical engineering, 2, 147-171.

2) Irshad, H., Veillard, A., Roux, L., \& Racoceanu, D. (2014). Methods for nuclei detection, segmentation, and classification in digital histopathology: a review - current status and future potential. IEEE reviews in biomedical engineering, 7, 97-114.

3) Hinojosa, S., Dhal, K. G., Elaziz, M. A., Oliva, D., \& Cuevas, E. (2018). Entropy-based imagery segmentation for breast histology using the Stochastic Fractal Search. Neurocomputing, 321, 201-215.

4) Liu, Q., Liu, P. X., Wang, Y., \& Leung, H. (2016). Semiparametric decolorization with Laplacian-based perceptual quality metric. IEEE Transactions on Circuits and Systems for Video Technology, 27(9), 1856-1868.

5) Neumann, L., Čadík, M., \& Nemcsics, A. (2007, June). An efficient perception-based adaptive color to gray transformation. In Proceedings of the Third Eurographics conference on Computational Aesthetics in Graphics, Visualization and Imaging (pp. 73-80). Eurographics Association.

6) Smith, K., Landes, P. E., Thollot, J., \& Myszkowski, K. (2008, April). Apparent greyscale: A simple and fast conversion to perceptually accurate images and video. In Computer Graphics Forum (Vol. 27, No. 2, pp. 193-200). Oxford, UK: Blackwell Publishing Ltd.

7) Gooch, A. A., Olsen, S. C., Tumblin, J., \& Gooch, B. (2005, July). Color2gray: salience-preserving color removal. In ACM
Transactions on Graphics (TOG) (Vol. 24, No. 3, pp. 634-639). ACM.

8) Lu, C., Xu, L., \& Jia, J. (2012, November). Real-time contrast preserving decolorization. In SIGGRAPH Asia 2012 Technical Briefs (p. 34). ACM.

9) Liu, Q., Liu, P. X., Xie, W., Wang, Y., \& Liang, D. (2015). GcsDecolor: gradient correlation similarity for efficient contrast preserving decolorization. IEEE Transactions on Image Processing, 24(9), 2889-2904.

10) Nafchi, H. Z., Shahkolaei, A., Hedjam, R., \& Cheriet, M. (2017). CorrC2G: Color to gray conversion by correlation. IEEE Signal Processing Letters, 24(11), 1651-1655.

11) MATLAB and Image Processing Toolbox Release 2012b, The MathWorks, Inc., Natick, Massachusetts, United States.

12) Xiong, J., Lu, H., Liu, Q., \& Xu, X. (2018). Parametric ratiobased method for efficient contrast-preserving decolorization. Multimedia Tools and Applications, 77(12), 15721-15745.

13) Du, H., He, S., Sheng, B., Ma, L., \& Lau, R. W. (2014). Saliency-guided color-to-gray conversion using region-based optimization. IEEE Transactions on Image Processing, 24(1), 434-443.

14) Jin, Z., Li, F., \& Ng, M. K. (2014). A variational approach for image decolorization by variance maximization. SIAM Journal on Imaging Sciences, 7(2), 944-968.

15) Labati, R. D., Piuri, V., \& Scotti, F. (2011, September). AllIDB: The acute lymphoblastic leukemia image database for image processing. In 2011 18th IEEE International Conference on Image Processing (pp. 2045-2048). IEEE.

16) Dhal, K. G., Ray, S., Das, S., Biswas, A., \& Ghosh, S. HuePreserving and Gamut Problem-Free Histopathology Image Enhancement. Iranian Journal of Science and Technology, Transactions of Electrical Engineering, 1-28.

17) Dhal, K. G., Fister Jr, I., Das, A., Ray, S., \& Das, S (2018) Breast Histopathology Image Clustering using Cuckoo Search Algorithm. StuCoSReC. 5th Student Computer Science Research Conference, 47-54.

18) Beghdadi, A., \& Le Negrate, A. (1989). Contrast enhancement technique based on local detection of edges. Computer Vision, Graphics, and Image Processing, 46(2), 162-174.

19) Dhal, K. G., Ray, S., Das, A., \& Das, S. (2018). A Survey on Nature-Inspired Optimization Algorithms and Their Application in Image Enhancement Domain. Archives of Computational Methods in Engineering, 1-32.

20) Ma, K., Zhao, T., Zeng, K., \& Wang, Z. (2015). Objective quality assessment for color-to-gray image conversion. IEEE Transactions on Image Processing, 24(12), 4673-4685. 